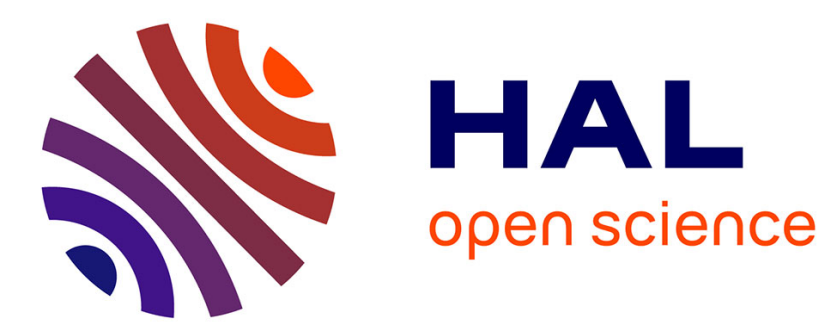

\title{
A semi-implicit level set method for multiphase flows and fluid-structure interaction problems
}

\author{
Georges-Henri Cottet, Emmanuel Maitre
}

\section{To cite this version:}

Georges-Henri Cottet, Emmanuel Maitre. A semi-implicit level set method for multiphase flows and fluid-structure interaction problems. Journal of Computational Physics, 2016, 314, pp.80-92. 10.1016/j.jcp.2016.03.004 . hal-01188443

\section{HAL Id: hal-01188443 \\ https://hal.science/hal-01188443}

Submitted on 30 Aug 2015

HAL is a multi-disciplinary open access archive for the deposit and dissemination of scientific research documents, whether they are published or not. The documents may come from teaching and research institutions in France or abroad, or from public or private research centers.
L'archive ouverte pluridisciplinaire HAL, est destinée au dépôt et à la diffusion de documents scientifiques de niveau recherche, publiés ou non, émanant des établissements d'enseignement et de recherche français ou étrangers, des laboratoires publics ou privés. 


\title{
A semi-implicit level set method for multiphase flows and fluid-structure interaction problems
}

\author{
Georges-Henri Cottet and Emmanuel Maitre \\ Univ. Grenoble Alpes and CNRS, Laboratoire Jean Kuntzmann, Grenoble, France. \\ e-mail: georges-henri.cottet@imag.fr; emmanuel.maitre@imag.fr
}

\begin{abstract}
In this paper we present a novel semi-implicit time-discretization of the level set method introduced in [8] for fluid-structure interaction problems. The idea stems form a linear stability analysis derived on a simplified one-dimensional problem. The semi-implicit scheme relies on a simple filter operating as a post-processing on the level set function. It applies to multiphase flows driven by surface tension as well as to fluid-structure interaction problems. The semi-implicit scheme avoids the stability constraints that explicit scheme need to satisfy and reduces significantly the computational cost. It is validated through comparisons with the original explicit scheme and refinement studies on two and three-dimensional membranes.
\end{abstract}

\section{Introduction}

Level set methods are classical methods to capture Lagrangian interfaces moving in complex flows [21]. In these methods the interface is implicitly given by the zero level of a function which is solution to an advection equation. Level set methods offer an alternative to interface capturing methods where interfaces are explicitly parametrized and followed along the flow. Compared to interface capturing methods one advantage of level set methods is their simplicity, since they only rely on the discretization of an advection equation, and their ability to follow topology changes of the interfaces. The accuracy of these methods, and in particular the fulfillment of conservation properties, rely on that of the discretization method used to solve the advection equation.

In Computational Fluid Dynamics, since the pioneering works $[29,6]$ level set methods are mostly applied to compute multiphase flows. In that case the level set function is used to compute geometrical information on the interface, typically the local normal and curvature, which allows to express capillary forces. Surface advection can be coupled to the interface motion [32]. Several recent works, often combining level set methods with Volume-of-Fluid (VOF) methods, have in particular been devoted to improve the accuracy of the curvature evaluation $[24,14]$ or to couple .

Level set methods have been extended to account for elastic forces in fluid-structure interaction problems involving elastic membranes in three dimensions. Originally devised for membrane elasticity given by area changes in incompressible flows [7, 8], these methods were further extended to the case of compressible flows [1] and to handle general 3D elasticity [9]. They were implemented for biological applications in the context of three-dimensional finite-difference methods $[17,5]$ or two-dimensional finite-element methods [25]. The case of elastic membranes with elasticity governed by shear and area change was recently studied in [19].

Whether based on front capturing Immersed Boundary Methods [22, 23] or level set methods, stability issues often arise in the calculation of multiphase flows and fluid-structure interaction problems and impose some strong constraints on the size of the time-steps.

The stability of the Immersed Boundary Method is well known to be sensitive to the stiffness of the elastic force applied on the structure when it is discretized in an explicit fashion. In order to remove the time step restrictions, several works have been dedicated to the derivation and study of implicit schemes [31, 18, 28, 27, 20]. However these schemes seem to be of limited practical interest because of the large computational cost related to the iterative resolution of a strongly non-linear coupling at each time step. The definition of semi-implicit or approximate implicit schemes leads to more realistic methods (see [31, 28, 27, 10, 11] and [2] and the references therein for details about such schemes). The search for an unconditionally stable scheme for curvature forces in an immersed boundary framework was already addressed specifically for the calculation of surface tension $[15,30]$.

There exist several ways to carry out the stability analysis of time-discretization schemes of fluid-structure coupling methods. Among them is the control of discrete energy conservation [2, 20], which is based on the 
corresponding physical property of the continuous underlying models. Another way is to perform a linear analysis near an equilibrium state of the system. In [28, 27] this approach enables the authors to show that the instability is in particular enhanced by the combination of a small fluid viscosity and a strong elastic force.

In the unpublished work [3] stability conditions on the time step for explicit and implicit discretization, schemes were derived for a linearized version of a simplified one-dimensional version of the level set model [8]. Despite the simplifications involved in this analysis, this ad-hoc model enabled us to exhibit relationships between the fluid viscosity, the elastic force, and the numerical parameters. The results allowed to recover already known results obtained by Brackbill et al. [4] and Vigneaux [12, 13], depending on the physical parameters range. Like in these references, explicit schemes were shown to require time step values of the order of $\lambda \Delta x^{3 / 2}$, where $\lambda$ is the stiffness of the membrane, in fluid-structure interaction problems, or the surface tension in multiphase flows.

In the present work, we introduce a new semi-implicit scheme, which is linearly unconditionally stable and applies both to multiphase flows and to fluid-structure interaction problems. Our strategy consists of solving a diffusion equation to predict the interface position where curvature or elastic forces are computed.

An outline of this paper is as follows. In section 2 we recall the approach defined in [7, 8] to derive level set methods for immersed membranes. In section 3 we summarize the stability analysis performed in [3]. In section 4 we derive our semi-implicit scheme and shows its unconditional stability. Section 5 is devoted to numerical illustrations of the stability and accuracy of this semi-implicit scheme, both for capillary flows and fluid-structure interactions. Finally, we draw in section 6 some conclusions and indicate directions for future work.

\section{Level set methods for fluid-structure interaction}

We recall here the derivation of Eulerian level set methods for the fluid structure interaction resulting form an elastic membrane immersed in and incompressible fluid $[7,8]$. We consider a computational domain $\Omega$ in $\mathbb{R}^{d}$ with $d=2$ or $d=3$, filled with a viscous incompressible and homogeneous fluid of density $\rho>0$ and viscosity $\mu>0$, containing an elastic membrane $\Gamma_{e}(t)$. For simplicity here we assume that he membrane is massless, and external forces are neglected. We also assume that this membrane only reacts to area changes, but not to tangential shear. This is for instance the case for models of membrane cells considered in biophysics, such as phospholipidic bilayers.

We introduce a level set function $\phi$ initialized as the signed distance to the initial membrane $\Gamma_{e}(0)$, and such that :

$$
\Gamma_{e}(t)=\{x \in \Omega, \phi(t, x)=0\}, \forall t \in[0, T] .
$$

In the case when the tangential shear does not create a stress, it can be shown that the surface elastic energy for this membrane can be approached by the volume energy:

$$
\mathcal{E}_{\varepsilon}(\phi)=\int_{\Omega} E_{e}(|\nabla \phi|) \frac{1}{\varepsilon} \zeta\left(\frac{\phi}{\varepsilon}\right) d x
$$

where $\varepsilon>0$ is a small numerical parameter related to interface smoothing, and $\zeta$ a mollifying 1D function of total integral equal to 1 . The elastic force that results from this energy is given by

$$
F_{\varepsilon}[\phi]=\left\{\mathbb{P}_{\nabla \phi^{\perp}}\left(\nabla\left[E^{\prime}(|\nabla \phi|)\right]\right)-E^{\prime}(|\nabla \phi|) \kappa(\phi) \frac{\nabla \phi}{|\nabla \phi|}\right\}|\nabla \phi| \frac{1}{\varepsilon} \zeta\left(\frac{\phi}{\varepsilon}\right),
$$

where $\mathbb{P}_{\nabla \phi^{\perp}}:=\mathbb{I}-\frac{\nabla \phi \otimes \nabla \phi}{|\nabla \phi|^{2}}$ is the projector on the tangent plane, and $\nu_{e}$ and $\kappa(\phi)$ are respectively the stiffness coefficient and the mean curvature of $\Gamma_{e}(t)$. The Eulerian level set model then reads:

$$
\left\{\begin{array}{l}
\rho\left(\partial_{t} u+u \cdot \nabla u\right)+\nabla p-\mu \Delta u=F_{\varepsilon} \\
\operatorname{div} u=0 \\
\partial_{t} \phi+u \cdot \nabla \phi=0
\end{array}\right.
$$

We will focus on two particular cases of stress-strain relationship, corresponding to quadratic or linear energy:

$$
E_{e}^{\prime}(r)=\nu_{e}(r-1) \text { or } E_{e}^{\prime}(r)=\nu_{e}
$$

The first case corresponds to linear elasticity while in the second case, there is no tangential component in the force $F_{\varepsilon}$ which reduces to the usual capillary force on the interface between two fluids. 


\section{A stability analysis for explicit and implicit coupling schemes}

From now on we assume without a loss of generality that the cut-off function $\zeta$ is an even function, with support in $[-1,+1]$, and that $\zeta(r)$ is a decreasing function of $r>0$. For simplicity we will also set $\rho=1$, but the general case can be recovered by substituting $\mu$ and $\nu_{e}$ in by $\mu / \rho$ and $\nu_{e} / \rho$ in (3) and (4)

We summarize in this section the analysis performed in [3]. We consider the case of a linear potential. A one-dimensional version of the model (3) is given by :

$$
\left\{\begin{array}{l}
\partial_{t} u+u \partial_{x} u-\mu \partial_{x x}^{2} u=-\nu_{e} \partial_{x x}^{2} \phi \partial_{x} \phi \frac{1}{\varepsilon} \zeta\left(\frac{\phi}{\varepsilon}\right), \\
\partial_{t} \phi+u \partial_{x} \phi=0 .
\end{array}\right.
$$

We investigate the stability of this system around the trivial stationary solution $(\bar{u}, \bar{\phi})$ given by $\bar{u}(x)=0, \bar{\phi}(x)=$ $x$. The linearized model is:

$$
\left\{\begin{array}{l}
\frac{\partial u}{\partial t}-\mu \frac{\partial^{2} u}{\partial x^{2}}=-\frac{\nu_{e}}{\varepsilon} \frac{\partial^{2} \phi}{\partial x^{2}} \text { on }[0, T] \times \mathbb{R}, \\
\partial_{t} \phi+u=0 \text { on }[0, T] \times \mathbb{R}, \\
u(0, x)=f(x), \phi(0, x)=g(x) \text { on } \mathbb{R} .
\end{array}\right.
$$

Equivalently, $\phi$ verifies a strongly damped wave equation:

$$
\frac{\partial^{2} \phi}{\partial t^{2}}-\mu \frac{\partial^{3} \phi}{\partial x^{2} \partial t}-\frac{\nu_{e}}{\varepsilon} \frac{\partial^{2} \phi}{\partial x^{2}}=0 .
$$

We consider a classical centered discretization of second order derivatives, with grid size $\Delta x$. The time step is denoted by $\Delta t$ and we set $t_{n}=n \Delta t$.

The natural explicit scheme consists of first computing the velocity at time $t_{n+1}$ by solving the Navier-Stokes equations in $\left[t_{n}, n+1\right]$ with the value of $\phi$ at time $t_{n}$, and then use this velocity to advect the level-set function. This gives the following equations:

$$
\left\{\begin{array}{l}
\frac{u_{j}^{n+1}-u_{j}^{n}}{\Delta t}-\mu \frac{u_{j+1}^{n+1}-2 u_{j}^{n+1}+u_{j-1}^{n+1}}{(\Delta x)^{2}}=-\frac{\nu_{e}}{\varepsilon} \frac{\phi_{j+1}^{n}-2 \phi_{j}^{n}+\phi_{j-1}^{n}}{(\Delta x)^{2}}, \\
\frac{\phi_{j}^{n+1}-\phi_{j}^{n}}{\Delta t}+u_{j}^{n+1}=0, \\
u_{j}^{0}=f_{j}, \phi_{j}^{0}=g_{j} .
\end{array}\right.
$$

We now classically assume that $\varepsilon$ is proportional to $\Delta x$ and for simplicity we set $\varepsilon=\Delta \mathrm{x}$. The stability condition obtained in [3] for this scheme then reads:

$$
\Delta \mathrm{t} \lesssim \frac{\mu \Delta \mathrm{x}+\max \left(\mu, \sqrt{\nu_{e} \Delta \mathrm{x}}\right) \Delta \mathrm{x}}{\nu_{e}} .
$$

In the limit of inviscid fluids, for which $\mu=0$, or for high Reynolds numbers of surface tension coefficient, we recover the condition of Brackbill et al. [4], namely

$$
\Delta \mathrm{t} \lesssim \frac{\Delta \mathrm{x}^{\frac{3}{2}}}{\sqrt{\nu_{e}}}
$$

Alternatively when the fluid is viscous enough and/or the surface tension coefficient small enough, then the condition reduces to the one derived by Galusinski and Vigneaux [12],

$$
\Delta \mathrm{t} \lesssim \mu \frac{\Delta \mathrm{x}}{\nu_{e}}
$$

This kind of mixed stability condition was also obtained in [13], but our linear analysis seems more elementary, and extends to the following implicit scheme :

$$
\left\{\begin{array}{l}
\frac{u_{j}^{n+1}-u_{j}^{n}}{\Delta t}-\mu \frac{u_{j+1}^{n+1}-2 u_{j}^{n+1}+u_{j-1}^{n+1}}{(\Delta x)^{2}}=-\frac{\nu_{e}}{\varepsilon} \frac{\phi_{j+1}^{n+1}-2 \phi_{j}^{n+1}+\phi_{j-1}^{n+1}}{(\Delta x)^{2}}, \\
\frac{\phi_{j}^{n+1}-\phi_{j}^{n}}{\Delta t}+u_{j}^{n+1}=0 \\
u_{j}^{0}=f_{j}, \phi_{j}^{0}=g_{j} .
\end{array}\right.
$$


For this scheme one can prove unconditional stability.

Observe that for the real fluid-structure coupling problem, the implicit scheme corresponding to (11) is nearly intractable since it would involve the resolution of a fully nonlinear problem coupling the Navier-Stokes equations with nonlinear source term and a transport equation. By contrast, we propose in next section a semi-implicit scheme which is unconditionally stable at negligible additional cost compared to the explicit scheme. In [3] the validity and limitations of the above analysis are illustrated by numerical experiments on oscillating droplets.

\section{A semi-implicit time-discretization}

To derive our semi-implicit method, we first start with the $1 \mathrm{D}$ toy problem that allowed to analyze the linear stability of the explicit and implicit schemes. We then consider the case of flows driven by surface tension, which corresponds to a linear elastic potential, and finally the general level set model.

\subsection{Linear model}

We start from the system (3) and its implicit time-discretization coupled with a centered finite-difference approximation of the Laplace operator (11). We can rewrite the first equation of (11) as

$$
u^{n+1}=u^{n}-\frac{\nu_{e} \Delta \mathrm{t}}{\epsilon} \Delta_{h} \phi^{n+1}+\mu \Delta \mathrm{t} \Delta_{h} u^{n+1},
$$

where $\Delta_{h} \phi_{j}=\left(\phi_{j-1}-2 \phi_{j}+\phi_{j+1}\right) \Delta \mathrm{x}^{-2}$. We then keep the two first terms in the right hand side above and use that as an approximation of $u^{n+1}$ in the second equation of (11). We obtain a prediction $\psi^{n+1}$ of $\phi^{n+1}$ as the solution of

$$
\frac{\psi^{n+1}-\phi^{n}}{\Delta \mathrm{t}}-\frac{\nu_{e} \Delta \mathrm{t}}{\epsilon} \Delta_{h} \psi^{n+1}=-u^{n}
$$

This is a classical implicit Euler scheme for a diffusion equation, which is now uncoupled to the calculation of $u^{n+1}$. It can be solved in an efficient way on Cartesian grids using fast (FFT-based) Poisson solvers. We use this prediction to compute the source term of the first equation (in $u$ ) and then use the computed $u^{n+1}$ to advect $\phi^{n}$, which produces $\phi_{n+1}$. The semi-implicit scheme finally reads :

$$
\left\{\begin{array}{l}
\frac{\psi^{n+1}-\phi^{n}}{\Delta \mathrm{t}}-\frac{\nu_{e} \Delta \mathrm{t}}{\epsilon} \Delta_{h} \psi^{n+1}=-u^{n} \\
\frac{u^{n+1}-u^{n}}{\Delta t}-\mu \Delta_{h} u^{n+1}=-\frac{\nu_{e}}{\epsilon} \Delta_{h} \psi^{n+1} \\
\frac{\phi^{n+1}-\phi^{n}}{\Delta t}+u^{n+1}=0 \\
u_{j}^{0}=f_{j}, \phi_{j}^{0}=g_{j}
\end{array}\right.
$$

Proposition 1. The semi-implicit scheme (14) is unconditionally stable.

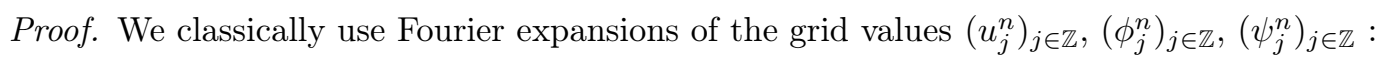

$$
u_{j}^{n}=\sum_{k \in \mathbb{Z}} \hat{u}^{n}(k) e^{2 i \pi k j \Delta \mathrm{x}}, \quad \phi_{j}^{n}=\sum_{k \in \mathbb{Z}} \hat{\phi}^{n}(k) e^{2 i \pi k j \Delta \mathrm{x}} \quad \psi_{j}^{n}=\sum_{k \in \mathbb{Z}} \hat{\psi}^{n}(k) e^{2 i \pi k j \Delta \mathrm{x}} .
$$

We set $\beta_{k}=\frac{4 \nu_{e} \Delta \mathrm{t}}{\Delta x^{3}} \sin ^{2}(\pi k \Delta \mathrm{x})$ (recall that we assumed $\left.\varepsilon=\Delta \mathrm{x}\right)$ and $\delta_{k}=\frac{4 \mu \Delta \mathrm{t}}{(\Delta x)^{2}} \sin ^{2}(\pi k \Delta \mathrm{x})$ and obtain from $(12),(13)$

$$
\begin{aligned}
\hat{\psi}^{n+1}(k)\left(1+\Delta \mathrm{t} \beta_{k}\right) & =\hat{\phi}^{n}(k)-\Delta \mathrm{t} \hat{u}^{n}(k) \\
\hat{u}^{n+1}(k)\left(1+\delta_{k}\right) & =\hat{u}^{n}(k)+\beta_{k} \hat{\psi}^{n+1}(k) \\
\hat{\phi}^{n+1} & =\hat{\phi}^{n}(k)-\Delta \mathrm{t} \hat{u}^{n+1}(k)
\end{aligned}
$$


or, equivalently, by substituting the first equation into the second one,

$$
A_{k}\left(\begin{array}{l}
\hat{u}^{n+1}(k) \\
\hat{\phi}^{n+1}(k)
\end{array}\right)=B_{k}\left(\begin{array}{c}
\hat{u}^{n}(k) \\
\hat{\phi}^{n}(k)
\end{array}\right)
$$

where

$$
A_{k}=\left(\begin{array}{cc}
1+\delta_{k} & 0 \\
\Delta \mathrm{t} & 1
\end{array}\right) \quad B_{k}=\left(\begin{array}{cc}
\frac{1}{1+\Delta \mathrm{t} \beta_{k}} & \frac{\beta_{k}}{1+\Delta \mathrm{t} \beta_{k}} \\
0 & 1
\end{array}\right)
$$

The eigenvalues $\lambda_{1}, \lambda_{2}$ of $A_{k}^{-1} B_{k}$ are the solutions of $\operatorname{det}\left(A_{k}^{-1} B_{k}-\lambda \mathbb{I}\right)=0$ or equivalently $\operatorname{det}\left(B_{k}-\lambda A_{k}\right)=0$ that is

$$
\left|\begin{array}{cc}
\frac{1}{1+\Delta \mathrm{t} \beta_{k}}-\lambda\left(1+\delta_{k}\right) & \frac{\beta_{k}}{1+\Delta \mathrm{t} \beta_{k}} \\
-\lambda \Delta \mathrm{t} & 1-\lambda
\end{array}\right|=0
$$

or equivalently

$$
\left|\begin{array}{cc}
1-\lambda \beta_{k}^{\prime} \delta_{k}^{\prime} & \beta_{k} \\
-\lambda \Delta \mathrm{t} & 1-\lambda
\end{array}\right|=0
$$

where we have set $\beta_{k}^{\prime}=1+\Delta \mathrm{t} \beta_{k}, \delta_{k}^{\prime}=1+\delta_{k}$. This gives the following equation verified by $\lambda_{1}, \lambda_{2}$ :

$$
\beta_{k}^{\prime} \delta_{k}^{\prime} \lambda^{2}-\lambda\left[1+\beta_{k}^{\prime} \delta_{k}^{\prime}-\Delta \mathrm{t} \beta_{k}\right]+1=0 .
$$

The product $\lambda_{1} \lambda_{2}$ is equal to $1 /\left(\beta_{k}^{\prime} \delta_{k}^{\prime}\right)<1$. If the eigenvalues are complex conjugate, their modulus is therefore less than 1. Their sum has the sign of $1+\beta_{k}^{\prime} \delta_{k}^{\prime}-\Delta \mathrm{t} \beta_{k}=2+\delta_{k}+\Delta \mathrm{t} \beta_{k} \delta_{k}>0$. As a result if $\lambda_{i}$ are real they are both positive. Moreover we can easily derive the following bounds

$$
\begin{aligned}
\lambda_{i} & \leq \frac{1+\beta_{k}^{\prime} \delta_{k}^{\prime}-\Delta \mathrm{t} \beta_{k}+\sqrt{\left(1+\beta_{k}^{\prime} \delta_{k}^{\prime}-\Delta \mathrm{t} \beta_{k}\right)^{2}-4 \beta_{k}^{\prime} \delta_{k}^{\prime}}}{2 \beta_{k}^{\prime} \delta_{k}^{\prime}} \\
& \leq \frac{1+\beta_{k}^{\prime} \delta_{k}^{\prime}+\sqrt{\left(1+\beta_{k}^{\prime} \delta_{k}^{\prime}\right)^{2}-4 \beta_{k}^{\prime} \delta_{k}^{\prime}}}{2 \beta_{k}^{\prime} \delta_{k}^{\prime}} \\
& \leq \frac{1+\beta_{k}^{\prime} \delta_{k}^{\prime}+\sqrt{\left(1-\beta_{k}^{\prime} \delta_{k}^{\prime}\right)^{2}}}{2 \beta_{k}^{\prime} \delta_{k}^{\prime}}=1 .
\end{aligned}
$$

The spectral radius of $A_{k}^{-1} B_{k}$ is therefore less than one and the scheme is unconditionnally stable.

One may wonder if the diffusion coefficient for $\phi$ used in (14) is optimal. If we carry out the same analysis using $\theta \beta_{k}$, with $\theta \leq 1$, instead of $\beta_{k}$ in (15) then we obtain a similar equation in $\lambda$ :

$$
\beta_{k}^{\prime} \delta_{k}^{\prime} \lambda^{2}-\lambda\left[1+\beta_{k}^{\prime} \delta_{k}^{\prime}-(2-\theta) \Delta \mathrm{t} \beta_{k}\right]+1-(1-\theta) \beta_{k} \Delta \mathrm{t}=0 .
$$

with the modification $\beta_{k}^{\prime}=1+\Delta \mathrm{t} \theta \beta_{k}$. A short computation shows that the product of roots is still less than one if $\theta \geq \frac{1}{2}$. For smaller values of $\theta$, instability could arise for $\beta_{k} \Delta t>\frac{2+\delta_{k}}{1-\left(2+\delta_{k}\right) \theta}$, which for inviscid flows gives $\Delta \mathrm{t}>\sqrt{\frac{2}{(1-2 \theta) \nu_{e}}} \Delta \mathrm{x}^{\frac{3}{2}}$. Even in the case $\theta \geq \frac{1}{2}$, the roots are not complex conjugate for $1-(1-\theta) \beta_{k} \Delta \mathrm{t}<0$, which can hold for some $k$ if $\Delta \mathrm{t}>\frac{\Delta \mathrm{x}^{\frac{3}{2}}}{\sqrt{(1-\theta) \nu_{e}}}$. Then their sum has the same sign as $2+\delta_{k}+\left(\theta\left(1+\delta_{k}\right)-1\right) \beta_{k} \Delta \mathrm{t}$ which could become negative for $\Delta$ t large enough provided that $\theta<\frac{1}{1+\delta_{k}}$. For high Reynolds fluids, that is when $\delta_{k} \ll 1$, or even for non viscous fluids where $\delta_{k}=0$, we therefore observe a change of behavior for $\theta<\theta_{c}$ with $\theta_{c}$ close or equal to 1 . This would lead to restrictions on time step of the same nature as in (9).

While this observation does not formally prove a change toward conditional stability, it at least gives some solid grounds to use as minimal diffusion coefficient the coefficient given in (14). Conversely, we will see in the sequel that taking a diffusion coefficient too large creates a gap between the filtered and the advected level set which can produce inconsistent results.

Remark 1. One can also consider the following variant, along the lines of the Crank-Nicolson method, of the semi-implicit scheme:

$$
\left\{\begin{array}{l}
\frac{u^{n+1}-u^{n}}{\Delta t}-\frac{\mu}{2} \Delta_{h} u^{n+1}-\frac{\mu}{2} \Delta_{h} u^{n}=-\frac{\nu_{e}}{\epsilon} \Delta_{h} \phi^{n+\frac{1}{2}} \\
\frac{\phi^{n+1}-\phi^{n}}{\Delta t}+\frac{1}{2}\left(u^{n+1}+u^{n}\right)=0 \\
u_{j}^{0}=f_{j}, \phi_{j}^{0}=g_{j}
\end{array}\right.
$$


Using the following approximations (well justified for small $\mu / \nu_{e}$ ):

$\phi^{n+\frac{1}{2}} \approx \frac{1}{2}\left(\phi^{n}+\phi^{n+1}\right)=\frac{1}{2}\left(\phi^{n}+\phi^{n}-\frac{\Delta \mathrm{t}}{2}\left(u^{n+1}+u^{n}\right)\right) \approx \phi^{n}-\frac{\Delta \mathrm{t}}{4}\left(u^{n}-\frac{\nu_{e} \Delta \mathrm{t}}{\epsilon} \Delta_{h} \phi^{n+\frac{1}{2}}+u^{n}\right)=\phi^{n}-\frac{\Delta \mathrm{t}}{2} u^{n}+\frac{\nu_{e} \Delta \mathrm{t}^{2}}{4 \epsilon} \Delta_{h} \phi^{n+\frac{1}{2}}$,

this provide another semi-implicit scheme which amounts to solving at each iteration the uncoupled system:

$$
\left\{\begin{array}{l}
\psi^{n+\frac{1}{2}}-\frac{\nu_{e} \Delta \mathrm{t}^{2}}{4 \epsilon} \Delta_{h} \psi^{n+\frac{1}{2}}=\phi^{n}-\frac{\Delta \mathrm{t}}{2} u^{n} \\
u^{n+1}-\frac{\mu \Delta \mathrm{t}}{2} \Delta_{h} u^{n+1}=u^{n}+\frac{\mu \Delta \mathrm{t}}{2} \Delta_{h} u^{n}-\frac{\nu_{e} \Delta \mathrm{t}}{\epsilon} \Delta_{h} \psi^{n+\frac{1}{2}} \\
\phi^{n+1}=\phi^{n}-\frac{\Delta \mathrm{t}}{2}\left(u^{n+1}+u^{n}\right) \\
u_{j}^{0}=f_{j}, \phi_{j}^{0}=g_{j}
\end{array}\right.
$$

The same analysis as above leads to the following matrices:

$$
A_{k}=\left(\begin{array}{cc}
1+\delta_{k} & 0 \\
\frac{\Delta \mathrm{t}}{2} & 1
\end{array}\right) \quad B_{k}=\left(\begin{array}{cc}
\frac{1-\Delta \mathrm{t} \beta_{k}}{1+\Delta \mathrm{t} \beta_{k}}-\delta_{k} & \frac{4 \beta_{k}}{1+\Delta \mathrm{t} \beta_{k}} \\
-\frac{\Delta \mathrm{t}}{2} & 1
\end{array}\right)
$$

with $\beta_{k}=\frac{\nu_{e} \Delta \mathrm{t}}{\Delta x^{3}} \sin ^{2}(\pi k \Delta \mathrm{x})$, and the equation on $\lambda$ :

$$
\delta_{k}^{\prime} \beta_{k}^{\prime} \lambda^{2}-2\left(1-\beta_{k} \Delta \mathrm{t}\right) \lambda+\left(1-\delta_{k}\right) \beta_{k}^{\prime}=0 .
$$

The product of the roots is thus $\frac{1-\delta_{k}}{1+\delta_{k}} \in[0,1]$. In the case of inviscid flows, this ratio is close to 1 and the discriminant is $-16 \beta_{k} \Delta \mathrm{t}<0$. The scheme is therefore unconditionally stable.

\subsection{The case of surface tension}

We now consider the fluid-structure interaction model when the elastic force is first reduced to a surface tension. This corresponds to $E^{\prime}(r)=\nu_{e}$ in (4) and the model reads:

$$
\left\{\begin{array}{l}
\partial_{t} u+u \cdot \nabla u-\mu \Delta u+\nabla p=-\nu_{e} \kappa(\phi) \nabla \phi \frac{1}{\varepsilon} \zeta\left(\frac{\phi}{\varepsilon}\right), \\
\operatorname{div} u=0 \\
\partial_{t} \phi+u \cdot \nabla \phi=0 .
\end{array}\right.
$$

A fully implicit time-discretization of the $(u, \phi)$ coupling in the above system would read

$$
\left\{\begin{array}{l}
\frac{u^{n+1}-u^{n}}{\Delta \mathrm{t}}=-\nu_{e} \kappa\left(\phi^{n+1}\right) \nabla \phi^{n+1} \frac{1}{\varepsilon} \zeta\left(\frac{\phi^{n+1}}{\varepsilon}\right)+R\left(u^{n}, u^{n+1}\right), \\
\operatorname{div} u^{n+1}=0, \\
\frac{\phi^{n+1}-\phi^{n}}{\Delta \mathrm{t}}+u^{n+1} \cdot \nabla \phi^{n+1}=0,
\end{array}\right.
$$

where $R\left(u^{n}, u^{n+1}\right)$ contains the time discretization of the inertial and diffusion terms in the Navier-Stokes equations. We further assume that $\phi$ is a signed distance function. In that case we have $\kappa(\phi)=\Delta \phi$. To derive our semi-implicit method we replace $\zeta\left(\phi^{n+1} / \varepsilon\right)$ by is maximal value that we will assume without loss of generality equal to 1 . If we discard the term $R$ in the right hand side of the equation for $u$ in (21) we obtain the following $O(\Delta \mathrm{t})$ approximation of $u^{n+1}$

$$
\tilde{u}^{n+1}=u^{n}-\frac{\nu_{e}}{\varepsilon} \Delta t \Delta \phi^{n+1} \nabla \phi^{n+1} .
$$

Inserting this expression in the advection equation for $\phi$ in (21) we obtain

$$
\frac{\tilde{\phi}_{j}^{n+1}-\phi_{j}^{n}}{\Delta t}-\frac{\nu_{e}}{\varepsilon} \Delta t \Delta \tilde{\phi}^{n+1}=-u^{n} \cdot \nabla \phi^{n} .
$$

The complete semi-implicit time-discretization scheme is thus given at each time step by the following sub-steps: 
Step 1: implicit diffusion on $\phi$

$$
\frac{\tilde{\phi}^{n+1}-\phi^{n}}{\Delta t}-\frac{\nu_{e}}{\varepsilon} \Delta t \Delta \tilde{\phi}^{n+1}=-u^{n} \cdot \nabla \phi^{n}
$$

Step 2: explicit time discretization of the Navier-Stokes equation :

$$
\frac{u^{n+1}-u^{n}}{\Delta t}-\mu \Delta u^{n+1}+u^{n} \cdot \nabla u^{n}+\nabla p=-\nu_{e} \kappa\left(\tilde{\phi}^{n+1}\right) \nabla \tilde{\phi}^{n+1} \frac{1}{\varepsilon} \zeta\left(\frac{\tilde{\phi}^{n+1}}{\varepsilon}\right) ; \operatorname{div} u^{n+1}=0
$$

Step 3: explicit advection for $\phi$ :

$$
\frac{\phi^{n+1}-\phi^{n}}{\Delta t}+u^{n+1} \cdot \nabla \phi^{n}=0
$$

This system has to be complemented by the appropriate boundary conditions for the velocity and level set functions. Like in the previous section, the semi-implicit character of the scheme only relies on an implicit time-stepping of a linear diffusion equation.

Several remarks on this scheme are in order.

Equation (24) acts as a filtering on the advection equation. The diffusion coefficient is proportional to $\Delta \mathrm{t} / \varepsilon$. In the regime we have in view where the Navier-Stokes equation are dominated by inertia terms the time-step will be bound by CFL conditions. If the interface width is proportional to the grid size, as it is often the case in practice, this diffusion coefficient is thus of order 1.

Next, it is important to observe that the original advected equation is not modified which is essential to preserve volume and prevents interface smearing. The filtering on the level set function acts only as a postprocessing of $\phi$ used to calculate the surface tension. It interferes with the advection equation only indirectly through the computation of the velocity.

Finally one may also consider a semi-implicit scheme by omitting the right hand side of (24). Similarly, in the case when $\phi$ is not a signed distance function, one can expand the curvature, following [26], as:

$$
|\nabla \phi| \kappa(\phi)=\Delta \phi-\frac{\nabla \phi}{|\nabla \phi|} \cdot \nabla|\nabla \phi| .
$$

This expression gives an extra source term in the right hand side of (24) in step 1. These two options have been tested. They do not change significantly the results.

\subsection{The semi-implicit scheme in the general case}

We finally turn to the general case of an elastic interface immersed in an incompressible fluid. We recall the expression of the elastic force as it appears in the right hand side of the Navier-Stokes equation, expressed in terms of tangential and normal components:

$$
F_{\varepsilon}[\phi]=\left\{\mathbb{P}_{\nabla \phi^{\perp}}\left(\nabla\left[E^{\prime}(|\nabla \phi|)\right]\right)-E^{\prime}(|\nabla \phi|) \kappa(\phi) \frac{\nabla \phi}{|\nabla \phi|}\right\}|\nabla \phi| \frac{1}{\varepsilon} \zeta\left(\frac{\phi}{\varepsilon}\right)
$$

where $E^{\prime}(r)=\lambda(r-1)$ and $\mathbb{P}_{\nabla \phi^{\perp}}$ is the projector on the tangent plane. We proceed like in the previous section and start from an implicit time-stepping for the Navier-Stokes equation

$$
\left\{\begin{array}{l}
\frac{u^{n+1}-u^{n}}{\Delta \mathrm{t}}=F_{\varepsilon}\left[\phi^{n+1}\right]+R\left(u^{n}, u^{n+1}\right), \\
\operatorname{div} u^{n+1}=0, \\
\frac{\phi^{n+1}-\phi^{n}}{\Delta \mathrm{t}}+u^{n+1} \cdot \nabla \phi^{n+1}=0,
\end{array}\right.
$$

from what we deduce the following prediction of $u^{n+1}$ :

$$
\tilde{u}^{n+1}=u^{n}-\Delta \mathrm{t} F_{\varepsilon}\left[\phi^{n+1}\right] .
$$

Upon inserting this expression in the advection equation for $\phi$ one observes that the tangential component of the force does not give any contribution and we are left with

$$
\frac{\tilde{\phi}_{j}^{n+1}-\phi_{j}^{n}}{\Delta t}-E^{\prime}\left(\left|\nabla \phi^{n}\right|\right) \frac{\Delta t}{\varepsilon} \Delta \tilde{\phi}^{n+1}=-u^{n} \cdot \nabla \phi^{n} .
$$

The semi-implicit method therefore goes along the following sub-steps: 
Step 1: implicit diffusion on $\phi$

$$
\frac{\tilde{\phi}^{n+1}-\phi^{n}}{\Delta t}-E^{\prime}\left(\left|\nabla \phi^{n}\right|\right) \frac{\Delta t}{\varepsilon} \Delta \tilde{\phi}^{n+1}=-u^{n} \cdot \nabla \phi^{n}
$$

Step 2: explicit time discretization of the Navier-Stokes equation

$$
\frac{u^{n+1}-u^{n}}{\Delta t}-\mu \Delta u^{n+1}+u^{n} \cdot \nabla u^{n}+\nabla p=F_{\varepsilon}\left[\tilde{\phi}^{n+1}\right] ; \operatorname{div} u^{n+1}=0
$$

Step 3: explicit advection for $\phi$

$$
\frac{\phi^{n+1}-\phi^{n}}{\Delta t}+u^{n+1} \cdot \nabla \phi^{n}=0
$$

As in the case of free surface flows with surface tension, we emphasize the fact that the filtering step on $\phi$ acts only as a post-processing used to compute forces and does not interfere directly with the advection of the level set function.

Unlike in the previous cases, the diffusion equation in step 2 is not a constant coefficient equation. To use fast Poisson solvers one may either replace the coefficient $E^{\prime}\left(\left|\nabla \hat{\phi}^{n}\right|\right)$ by its maximal value, or split this term into two parts: its maximal value and the residual. The diffusion equation (30) is then replaced by

$$
\frac{\tilde{\phi}^{n+1}-\phi^{n}}{\Delta t}-\max E^{\prime}\left(\left|\nabla \phi^{n}\right|\right) \frac{\Delta t}{\varepsilon} \Delta \tilde{\phi}^{n+1}=\left[E^{\prime}\left(\left|\nabla \hat{\phi}^{n}\right|-\max E^{\prime}\left(\left|\nabla \phi^{n}\right|\right)\right] \frac{\Delta t}{\varepsilon} \Delta \phi^{n}-u^{n} \cdot \nabla \phi^{n}\right.
$$

for which a fast Poisson solver can be used. We observed in our tests that the first option, where the diffusion coefficient is fixed at its maximum value, is over-diffusive and does not produce consistent results. We will come back to this point in section 5.2. The discretization (32) was used in our 3D experiments, while in $2 \mathrm{D}$ we used the original diffusion equation (29) together with a Choleski factorization at each time step.

\subsection{Discretization scheme}

The incompressible Navier-Stokes equation was solved using a classical projection method over a staggered MAC mesh grid. For the Reynolds numbers considered in this paper, this solver did not introduce stability constraints beyond the stability conditions related to the coupling with the forcing term in the right hand side of the Navier-Stokes equation.

In our 2D calculation he Poisson equations involved in the projection step and the diffusion equation (29) on $\phi$ were solved directly using a Choleski factorization. In the $3 \mathrm{D}$ implementation, to reduce the computational cost, we used the decomposition (30) together with the constant coefficient Poisson solver of the FISHPACK library [?].

Finally, for the advection equation on $\phi$, a fifth order WENO scheme was used with a CFL equal to ??. When the time-step of the Navier-Stokes equation violated this CFL condition, sub-iterations were performed for the advection equation.

\section{Numerical tests}

\subsection{Test case 1: surface tension for a relaxing droplet}

Although our main interest in this work is the fluid-structure interaction problem, we first consider the case of a viscous droplet subject to surface tension. In this case the semi-implicit scheme amounts to the very simple diffusion equation (24) .

We consider an initial interface of elliptic shape, with axis of sizes 0.5 and 0.75 respectively. Under the effect of surface tension the droplet relaxes to a circle with the same area. The surface tension coefficient was taken equal to 1 . All tests were performed with a constant time step of 0.0025 , for the equation (25). The interface width was taken as $\varepsilon=6 d x$. Figure 1 compares for $N=256$ the evolution of the two axis of the ellipse obtained by the level set method in the case of semi-implicit and explicit scheme. For these parameters the semi-implicit scheme, unlike the explicit scheme, proves to be stable.

Although a more systematic study will be performed in the next section, we show in Figure 2 a first illustration of the numerical convergence of our semi-implicit scheme as the mesh size tends to zero. In this figure the evolution of the small and large axis is depicted depicted for $N=256$ and $N=512$. 

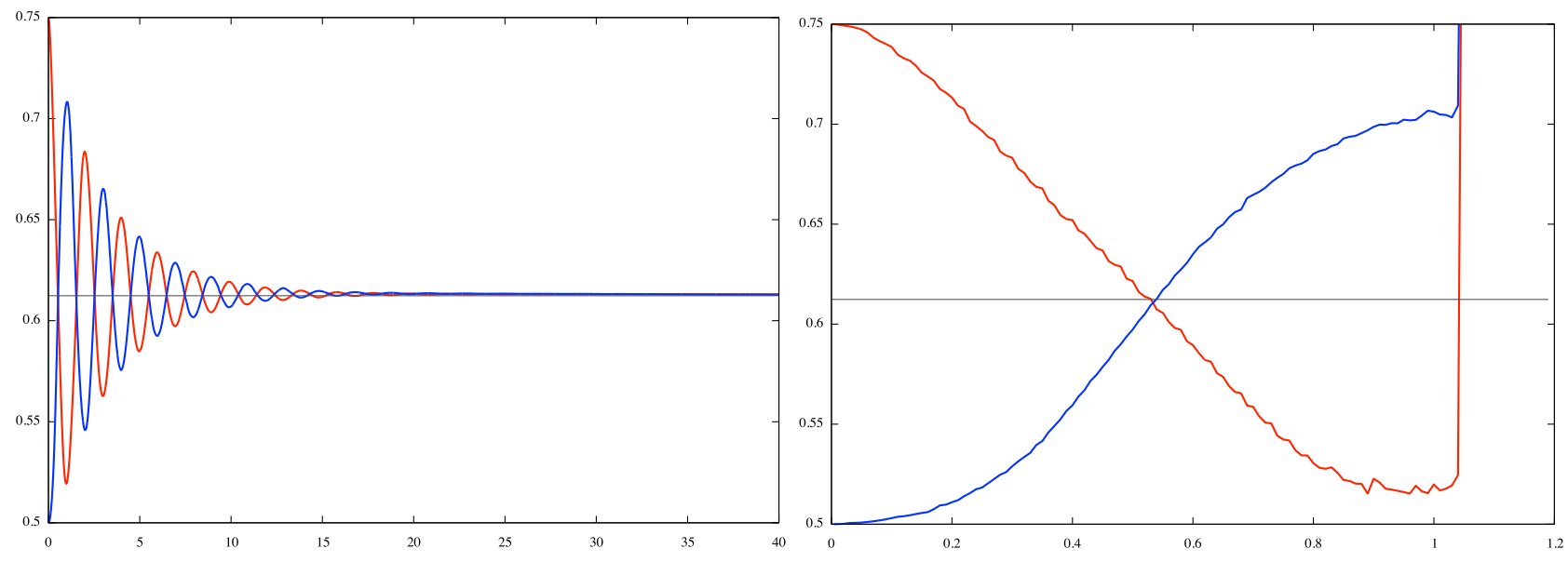

FIG 1. Oscillating droplet with $N=256$ and $\Delta \mathrm{t}=0.0025$. Time variation of radii obtained with the semi-implicit scheme (left picture) and the explicit scheme (right picture).

Our Eulerian model enjoys good conservation properties as it is based on a projection scheme on a staggered grid, which ensures at computer accuracy a zero divergence of the velocity field. To illustrate this feature we show in Figure 2 the volume loss inside the droplet for resolutions ranging from $N=64$ to $N=512$. One can see that the volume loss during the oscillations is kept below $1.5 \%$ for the coarsest resolution and below $0.1 \%$ for the higher resolution.
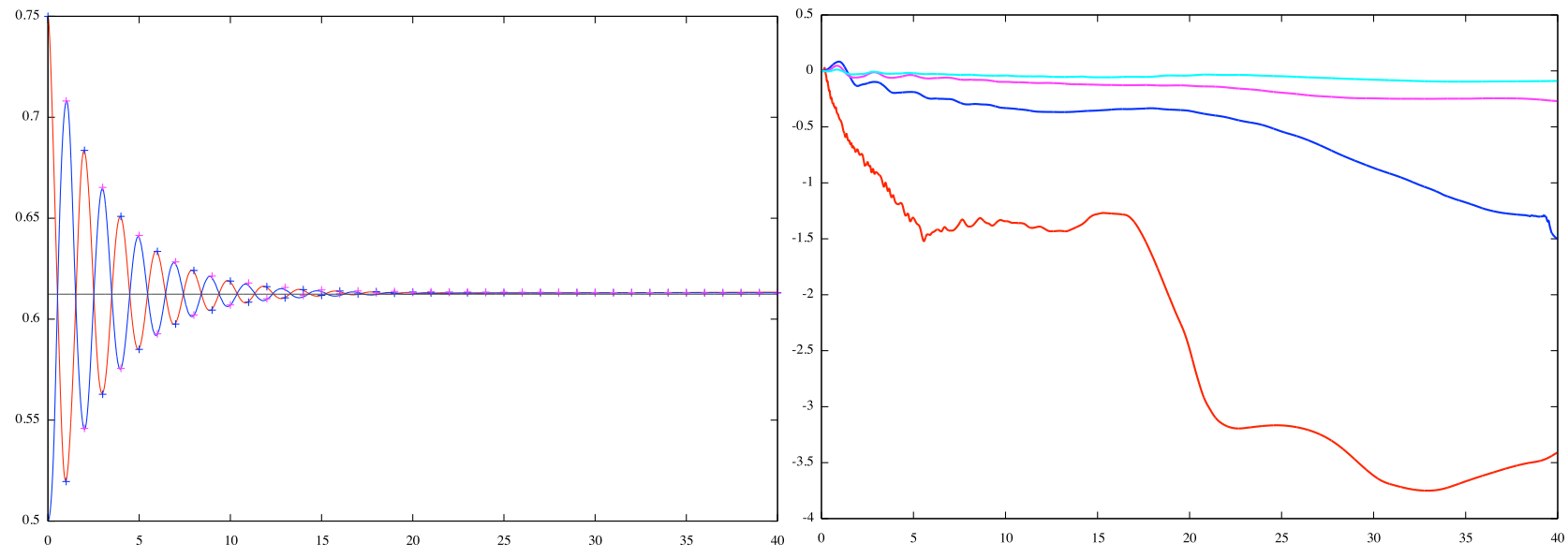

FIG 2. Oscillating droplet with the semi-implicit scheme. Left picture: Evolution of horizontal (blue) and vertical (red) radii, for $N=256$ (crosses) and $N=512$ (continuous lines). Right picture: Variation of volume inside the membrane (in \%) for $N=64$ (red), $N=128$ (blue), $N=256$ (magenta) and $N=512$ (cyan), with respect to time.

Note that in the present work we do not address the questions related to the accuracy of the computation of the surface tension (see for instance [24]). This is a delicate issue, in particular for high Reynolds number flows, due to the singularity of the curvature term. The behavior of the semi-implicit scheme regarding this issue will be investigated in a future work.

\subsection{Test case 2: an elastic interface in $\mathbb{R}^{2}$}

We now consider an elastic membrane, governed by the quadratic elastic potential (4). In this section and the next one, the value of the stiffness coefficient $\lambda$ is set to 10 . We use the same test case as in $[16,8]$. It consists of an elliptical membrane, with major and minor axis respectively equal to 0.75 and 0.5 , stretched from an 
equilibrium circular state. This corresponds to a uniform stretching rate of about 1.262. This case test, albeit simple, is in particular a good benchmark to verify the conservation properties of the method. Throughout this section, $\Delta t$ is the time-step used to solve the Navier-Stokes equation with the elastic force. As laready mentioned, depending on its value, sub-iterations may be used in the advection equation of the level set to satisfy the appropriate CFL condition.

We first address the already mentioned issue concerning the implementation of the variable-coefficient diffusion equation for the level set function. In Figure 3 we show the oscillations of the membrane when the value $\max \left|E^{\prime}\left(\nabla \phi^{n}\right)\right|$ is used at time $t_{n}$ as diffusion coefficient in (29), compared to the reference results obtained with the explicit scheme. The parameter were $N=128, \Delta \mathrm{t}=1.510^{-3}$ for the explicit scheme and $\Delta \mathrm{t}=810^{-3}$ for the semi-implicit scheme. One can see that this diffusion parameter does not produce correct oscillations. In particular the membrane does not relax to a circle. In the rest of the paper we therefore implemented the variable-coefficient diffusion equation, in tis original form (29) in 2D, and in its approximation (32) in 3D.

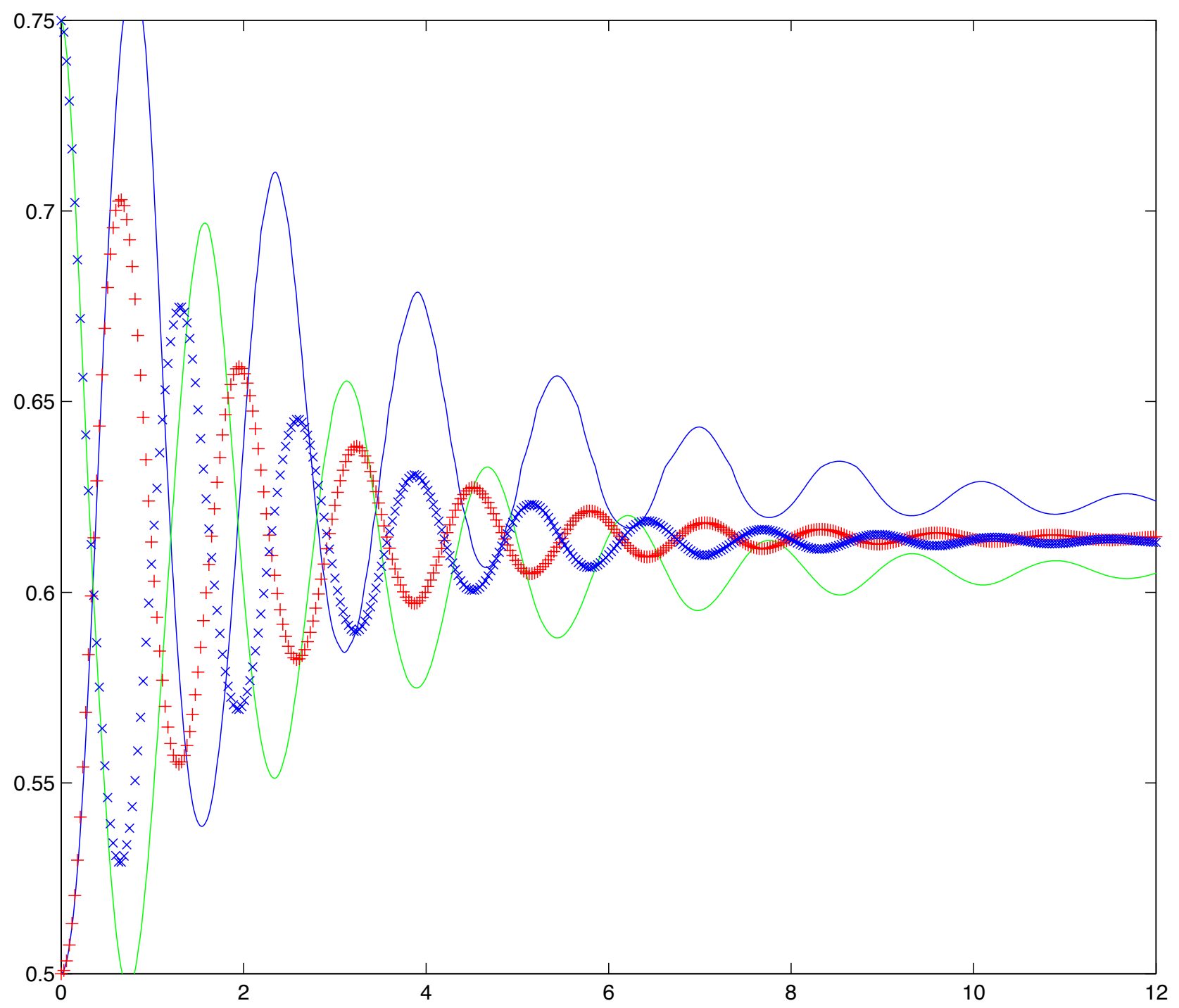

FIG 3. Relaxing elastic membrane. Semi-implicit scheme using the maximum of $E^{\prime}$ in the diffusion equation (29), for $N=128$. Solid lines are horizontal and vertical radii for the semi-implicit scheme with $\Delta \mathrm{t}=810^{-3}$ and dotted data corresponds to the reference explicit scheme with $\Delta \mathrm{t}=1.510^{-3}$.

We next investigate the stability properties of the semi-implicit scheme. For this purpose we performed two series of refinement studies. In the first series of test we kept for the semi-implicit scheme the same time-step for 
the level set equation for all grid resolutions, with a value $\Delta t=0.01$. In the second series of tests, the time-step chosen for the semi-implicit scheme was refined using a CFL condition of 0.25 while, for the explicit scheme, it had to be defined on the basis of the stability condition (9). Table 1 shows the values of the resulting time-steps for the explicit and semi-implicit schemes.

TABLE 1

Time step values used in the 2D experiments for the explicit and semi-implicit scheme.)

$\left|\begin{array}{l|c|c|}\mathrm{N} & \text { explicit } & \text { semi-implicit } \\ 64 & 3.510^{-3} & 10^{-2} \\ 128 & 1.510^{-3} & 8.10^{-3} \\ 256 & 6.510^{-4} & 4.10^{-3} \\ 512 & 2.10^{-4} & 2.10^{-3}\end{array}\right|$

Figure 4 shows the relaxation of the membrane, for resolutions corresponding to a number of grid-points in each direction ranging from $N=64$ to $N=256$, when the semi-implicit scheme is used with the time-step 0.01. These experiments confirm the stability of the semi-implicit but also show that for relatively high resolution and large time-steps the membrane undergoes in a primary stage non-physical oscillations. The reason is that, for large values of $\Delta t$, the elastic force, obtained from the filtered value of $\phi$, is applied at a location which differs significantly from the advected level set. Note however that if one is mostly interested in the equilibrium state, rather than in the details of the oscillations, large values of the time-step remain admissible.

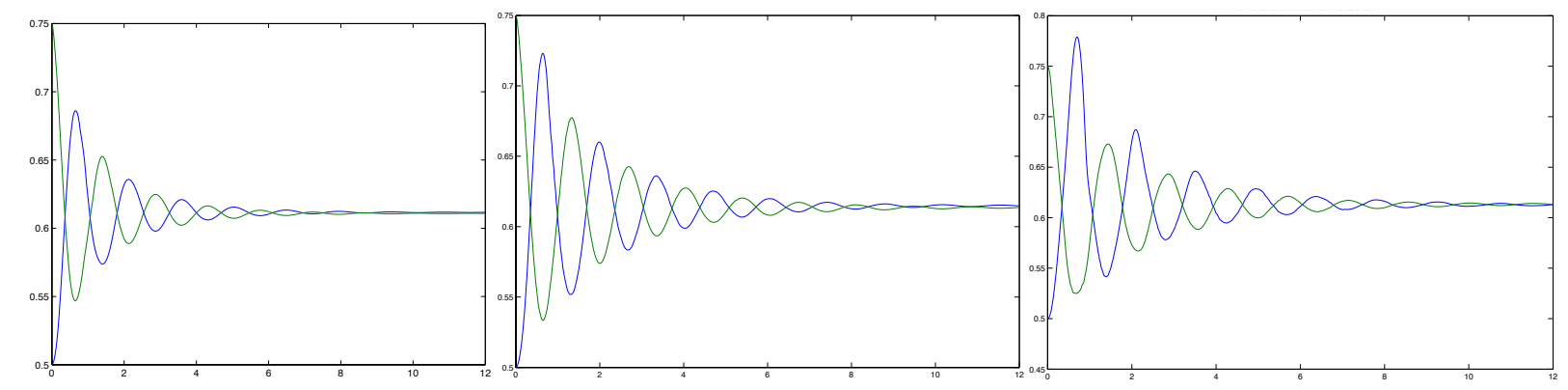

FIG 4. Relaxation of an elastic elliptical membrane. Time evolution of the large and small radii with the semi-implicit scheme for $\Delta \mathrm{t}=10^{-2}$ and, from left to right, $N=64,128,256$.

We now turn to the second series of refinement tests and compare in figure 5, for the time-steps given in Table 1, the results obtained with the explicit and semi-implicit schemes, for $N=128$ and $N=256$. On the left picture, for $N=128$, we used $\Delta \mathrm{t}=1.510^{-3}$ for the explicit scheme and $\Delta \mathrm{t}=810^{-3}$ for the semi-implicit scheme. On the right picture, we fixed $\Delta \mathrm{t}=6.510^{-4}$ for the explicit scheme and $\Delta \mathrm{t}=410^{-3}$ for the semiimplicit scheme. Given that for $N=256$ the time-step for the semi-implicit scheme is eight times smaller than for the explicit scheme, this experiment illustrates the gain offered by the semi-implicit method.

Figure 6 shows the oscillations of the membrane obtained with the semi-implicit scheme for $N=256$ and $N=512$, and the respective time-steps given in Table 1 , for $\varepsilon=1.5 d x$ and $\lambda=10$. It serves as a first illustration of the convergence properties of the semi-implicit scheme.

In order to measure in a more quantitative fashion the consistency of the semi-implicit scheme, Table 2 monitors the values of the horizontal radius at time $t=2$ for the explicit and implicit scheme for decreasing grid sizes corresponding to values of $N$ ranging from 64 to 1024 .

Convergence studies concerning the discretization of equations (3) are rather delicate. In the limit $\varepsilon \rightarrow 0$ the right hand side of (30) is singular and one expects that convergence requires to keep $\varepsilon>>\Delta x$. In the present study we have chosen to set $\varepsilon$ proportional to $\sqrt{\Delta x}$. For this study we have defined the result of the explicit scheme for $N=1024$ as the reference result to which we compare the results of the explicit and semi-implicit schemes at coarser resolutions. The results show that the semi-implicit scheme converges, albeit at a lower convergence rate than the explicit scheme. In this study the time-step was set along the lines of table 1 to satisfy the stability condition (9) for the explicit scheme and, for the semi-implicit scheme, a CFL condition with a CFL number equal to 0.25 . It is worthwhile to mention that for the finest resolution, with $N=1024$ 

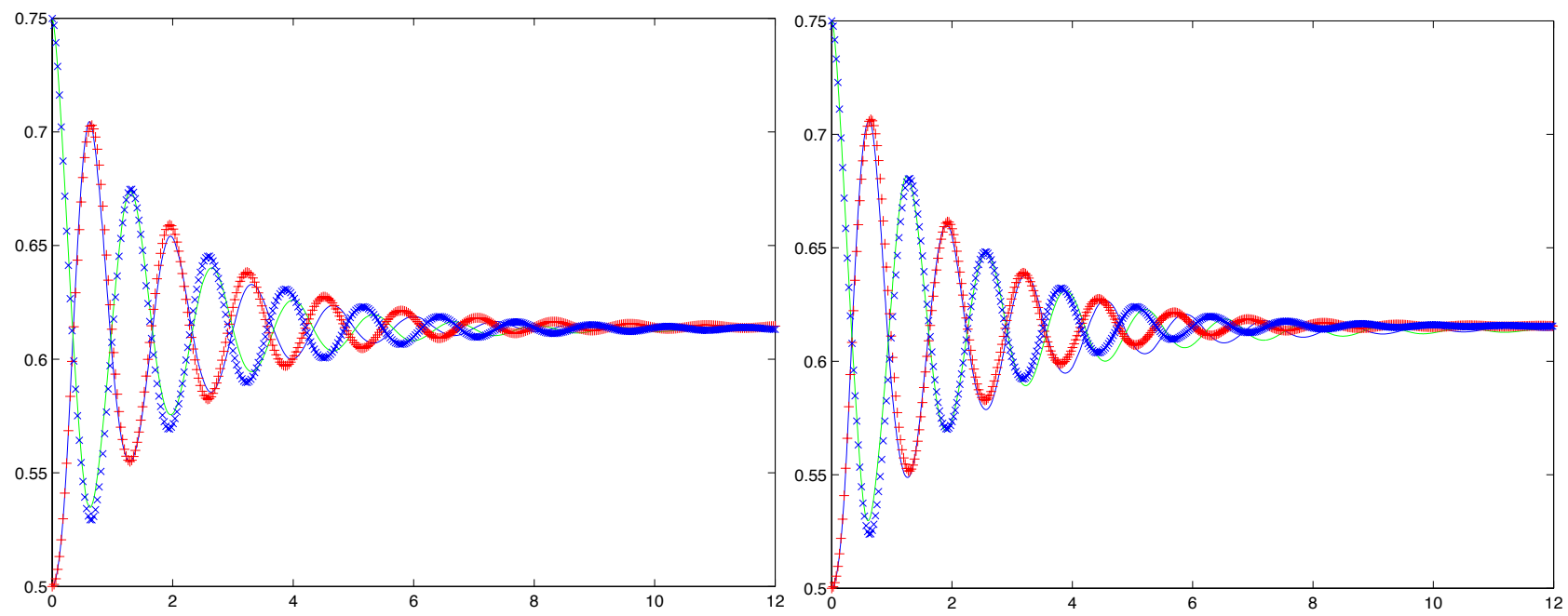

FIG 5. Relaxing 2D elastic membrane. Time variations of ellipse radii. Semi-implicit (continuous lines) vs explicit $(\times$ and + ) schemes for $N=128$ (left) and 256 (right).

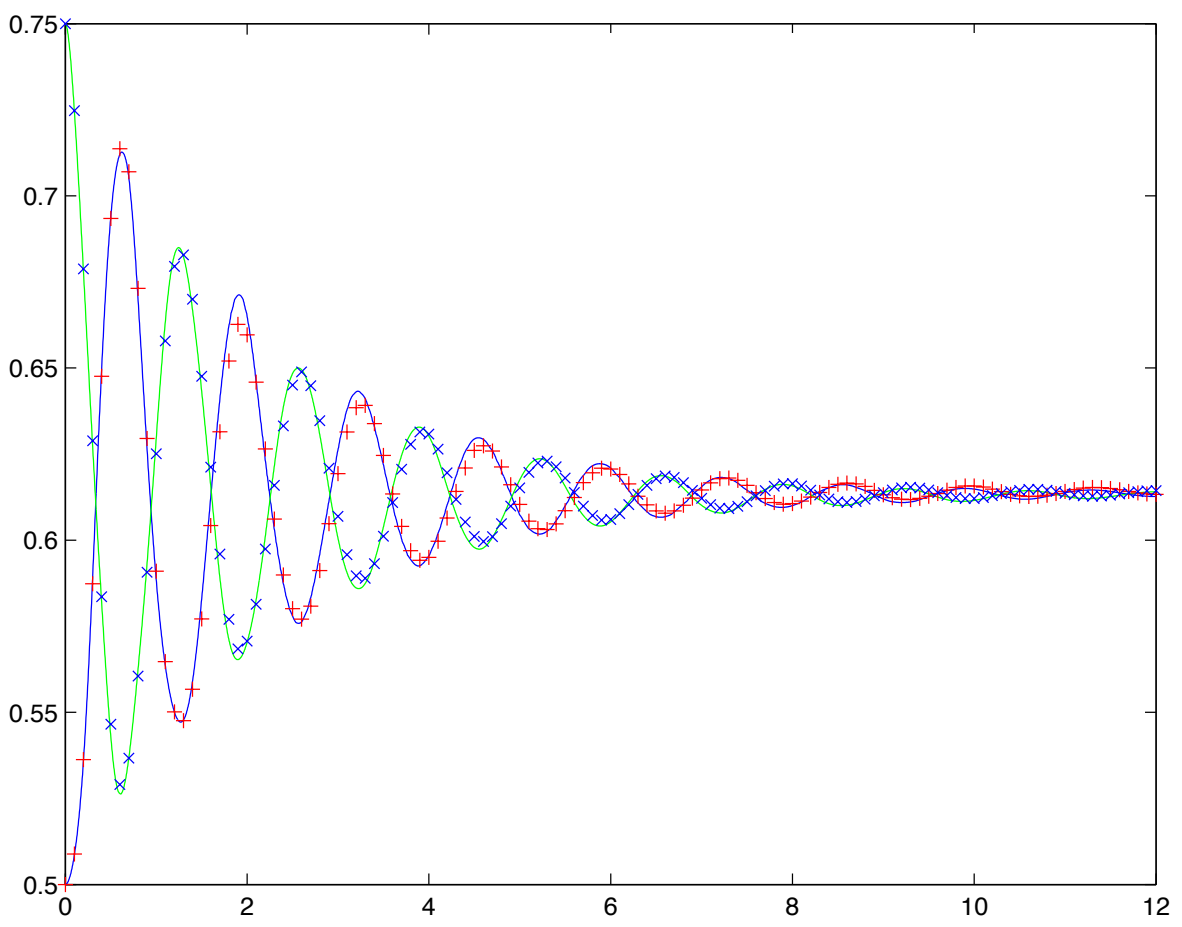

FIG 6. Relaxing 2D elastic membrane. Time variations of ellipse radii with the semi-implicit scheme for $N=256$ (dotted data) and 512 (continuous line). 
points in each direction, the cost/time-step for the explicit scheme ...

TABLE 2

Horizontal radii for explicit and semi-implicit schemes at time $t=2$ for the elastic membrane for grids ranging from $N=64$ to $N=1024$ grid points in each direction. $r_{e}$ (resp $r_{i}$ ) is the value obtained with the explicit (resp. semi-implicit) scheme. $\delta r_{e}$ (resp. $\left.\delta r_{i}\right)$ is the relative error with respect to the reference result (corresponding to the explicit scheme at the highest resolution) obtained with the explicit scheme (resp. semi-implicit) and $q_{e}$ (resp $q_{i}$ ) is the numerical order of convergence for the explicit (resp. implicit) scheme.

\begin{tabular}{|l|c|c|c|c|c|c|}
$\mathrm{N}$ & $r_{e}$ & $r_{i}$ & $\delta r_{e}$ & $q_{e}$ & $\delta r_{i}$ & $q_{i}$ \\
64 & 0.643587 & 0.63089 & 0.021 & & 0.041 & \\
128 & 0.652348 & 0.638246 & 0.0080 & 1.39 & 0.029 & 0.5 \\
256 & 0.654439 & 0.643734 & 0.0048 & 0.74 & 0.021 & 0.46 \\
512 & 0.656084 & 0.6473 & 0.0023 & 1.23 & 0.016 & 0.4 \\
1024 & 0.657617 & 0.653769 & 0. & & 0.006 & 1.41
\end{tabular}

\subsection{Test case 3: elastic interface in $\mathbb{R}^{3}$}

We finally consider the case of an immersed elastic surface inside a three-dimensional flow, endowed with an elastic law limited to area variation. The case of full membrane energy, including tangential shear, cannot be written in terms of $\phi$ only, and is therefore more intricate. It is studied in a forthcoming work [19]. We present below a test case to show the ability of our numerical method to deal with this $3 \mathrm{D}$ case. The elastic ellipse has radii of $0.8,0.6$ and 0.4 in the $x, y, z$ directions. We plot in Figure7 the variations of those three dimensions while the ellipse is relaxing to a sphere, using our semi-implicit scheme for $N=128$ and a time step of $2.510^{-3}$. Figure 8 depicts the values of the pressure in cross sections though the center of the sphere and $\mathrm{f}$ the elastic stretching along the membrane.
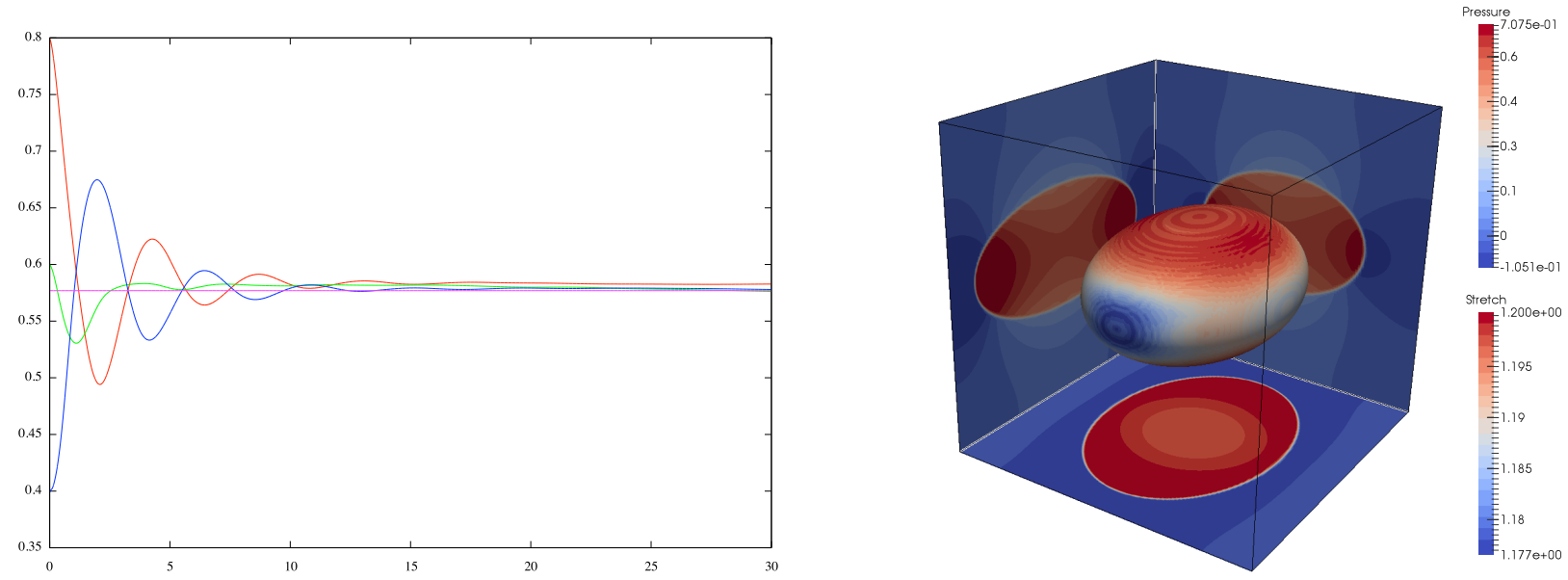

FIG 7. Relaxing 3D elastic membrane with the semi-implicit scheme for $N=128$ and $\Delta \mathrm{t}=2.510^{-3}$. Left picture: time variation of $x$-radius (red), $y$-radius (green) and $z$-radius (blue). Right picture : contours of stretching on the membrane and pressure value on cross-section through the center at $t=0.1$.

\section{Conclusion}

In this paper we have presented a novel semi-implicit scheme for the temporal discretization of level set methods in the context of multiphase flows or fluid-structure interaction problems. This method stems from the analysis of a linearized 1D model of such problems. Although based on a very simplified model, this analysis allows to recover classical stability constraints for explicit schemes in level set methods and to derive and prove unconditional linear stability for the semi-implicit scheme. 

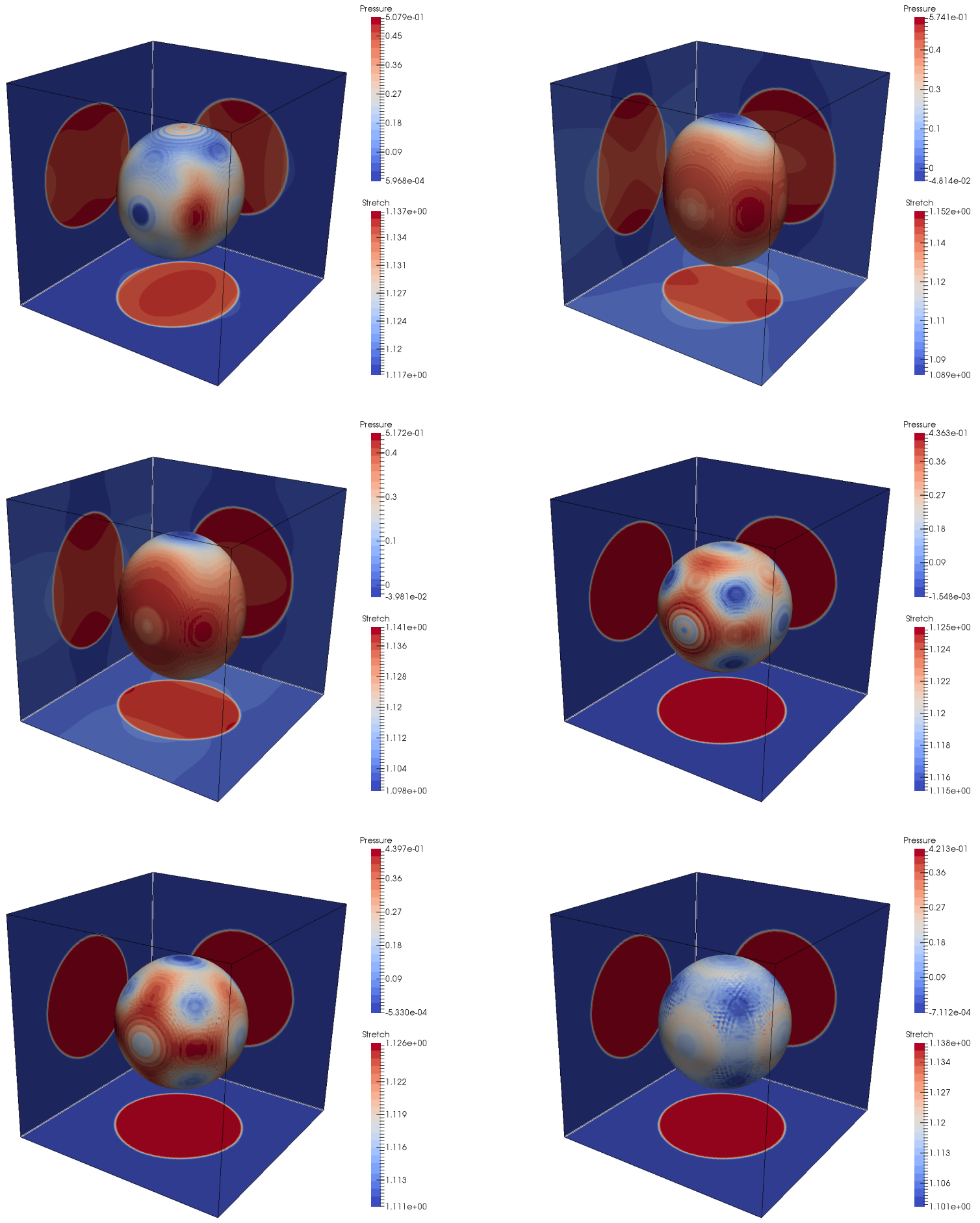

FIG 8. Relaxing $3 D$ elastic membrane with the semi-implicit scheme for $\Delta \mathrm{t}=2.510^{-3}$ and $N=128$. Contours of stretching on the membrane and pressure values on cross-section through the center at $t=1 ; 2 ; 2.5 ; 5 ; 15 ; 30$. 
The semi-implicit scheme is based on a variable coefficient diffusion equation for the level set equation, the coefficient of which is determined by the surface tension in the case of multiphase flows and by the elastic force in the case of the interaction of a fluid with an elastic membrane. This diffusion equation is used as a postprocessing to determine the values of the level set functions to be used for the computation of the the surface tension or the elastic force in the Navier-Stokes equation. It does not interfere with the advection equation that determines the location of the interface and therefore does not compromise the conservation properties of the overall method.

A series of comparisons with the explicit scheme and refinement studies demonstrate that, for moderate to high grid resolution, this method allows to significantly increase the time-step value, and thus reduce the computational cost, without deteriorating the desired accuracy. Future plans consist in particular of extending the method to level set models for generic fluid-structure interaction problems along the lines of [9, 19].

\section{References}

[1] J. Thomas Beale and John Strain. Locally corrected semi-lagrangian methods for stokes flow with moving elastic interfaces. Journal of Computational Physics, 227(8):3896 - 3920, 2008. 1

[2] Daniele Boffi, Lucia Gastaldi, and Luca Heltai. Numerical stability of the finite element immersed boundary method. Mathematical Models and Methods in Applied Sciences, 17(10):1479-1505, 2007.1

[3] Claire Bost, Georges-Henri Cottet, and Emmanuel Maitre. Linear stability analysis of a Level Set model of immersed elastic membrane. Unpublished. $\underline{\text { Preprint HAL }}$ http://hal.archives-ouvertes.fr/hal-00388528/PDF/stability.pdf, 2009. 2, 3, 4

[4] JU Brackbill, Douglas B Kothe, and C1 Zemach. A continuum method for modeling surface tension. Journal of computational physics, 100(2):335-354, 1992. 2, 3

[5] Didier Bresch, Thierry Colin, Emmanuel Grenier, Benjamin Ribba, and Olivier Saut. Computational modeling of solid tumor growth: the avascular stage. SIAM Journal on Scientific Computing, 32(4):23212344, 2010. 1

[6] Y.C. Chang, T.Y. Hou, B Merriman, and S. Osher. A level set formulation of eulerian interface capturing methods for incompressible fluid flows. Journal of Computational Physics, 124:449-464, 1996. 1

[7] Georges-Henri Cottet and Emmanuel Maitre. A level-set formulation of immersed boundary methods for fluid-structure interaction problems. Comptes Rendus Mathematique, 338(7):581-586, 2004. 1, 2

[8] Georges-Henri Cottet and Emmanuel Maitre. A level set method for fluid-structure interactions with immersed surfaces. Mathematical models and methods in applied sciences, 16(03):415-438, 2006. 1, 2, 9

[9] Georges-Henri Cottet, Emmanuel Maitre, and Thomas Milcent. Eulerian formulation and level set models for incompressible fluid-structure interaction. ESAIM: Mathematical Modelling and Numerical Analysis, 42(3):471-492, May 2008. 1, 15

[10] MA Fernández, J-F Gerbeau, and Celine Grandmont. A projection semi-implicit scheme for the coupling of an elastic structure with an incompressible fluid. International Journal for Numerical Methods in Engineering, 69(4):794-821, 2007. 1

[11] Miguel A Fernández, Jimmy Mullaert, and Marina Vidrascu. Explicit robin-neumann schemes for the coupling of incompressible fluids with thin-walled structures. Computer Methods in Applied Mechanics and Engineering, 267:566-593, 2013. 1

[12] Cédric Galusinski and Paul Vigneaux. Level-set method and stability condition for curvature-driven flows. Comptes Rendus Mathematique, 344(11):703-708, 2007. 2, 3

[13] Cédric Galusinski and Paul Vigneaux. On stability condition for bifluid flows with surface tension: Application to microfluidics. Journal of Computational Physics, 227(12):6140-6164, 2008. 2, 3

[14] M. Herrmann. A balanced force refined level set grid method for two-phase flows on unstructured flow solver grids. Journal of Computational Physics, 227:2674-2706, 2008. 1

[15] S Hysing. A new implicit surface tension implementation for interfacial flows. International Journal for Numerical Methods in Fluids, 51(6):659-672, 2006. 1

[16] Long Lee and Randall J LeVeque. An immersed interface method for incompressible navier-stokes equations. SIAM Journal on Scientific Computing, 25(3):832-856, 2003. 9

[17] Emmanuel Maitre, Thomas Milcent, Georges-Henri Cottet, Annie Raoult, and Yves Usson. Applications of level set methods in computational biophysics. Mathematical and Computer Modelling, 49(11-12):21612169, June 2009. 1 
[18] Anita A Mayo and Charles S Peskin. An implicit numerical method for fluid dynamics problems with immersed elastic boundaries. Contemporary Mathematics, 141:261-261, 1992. 1

[19] Thomas Milcent and Emmanuel Maitre. Eulerian model of immersed elastic surfaces with full membrane elasticity. Preprint HAL https://hal.archives-ouvertes.fr/hal-01078869, October 2014. to appear in Advances in Computational Mathematics. 1, 13, 15

[20] Elijah Paul Newren. Enhancing the immersed boundary method: stability, volume conservation, and implicit solvers. PhD thesis, The University of Utah, 2007. 1

[21] S. Osher and R. Fedkinw. Level Set Methods and Dynamic Implicit Surfaces. Springer-Verlag, New York, 2002. 1

[22] Charles S Peskin. Numerical analysis of blood flow in the heart. Journal of Computational Physics, 25(3):220 - 252, 1977. 1

[23] Charles S Peskin. The immersed boundary method. Acta numerica, 11:479-517, 2002. 1

[24] Stéphane Popinet. An accurate adaptive solver for surface-tension-driven interfacial flows. Journal of Computational Physics, 228:5838-5866, 2009. 1, 9

[25] Ricardo Ruiz-Baier, Alessio Gizzi, Simone Rossi, Christian Cherubini, Aymen Laadhari, Simonetta Filippi, and Alfio Quarteroni. Mathematical modelling of active contraction in isolated cardiomyocytes. Mathematical Medicine And Biology-A Journal Of The Ima, 31(3):259-283, 2014. 1

[26] Peter Smereka. Semi-implicit level set methods for curvature and surface diffusion motion. Journal of Scientific Computing, 19(1):439-456, 2003. 7

[27] John M Stockie and Brian R Wetton. Analysis of stiffness in the immersed boundary method and implications for time-stepping schemes. Journal of Computational Physics, 154(1):41-64, 1999. 1, 2

[28] John M Stockie and Brian TR Wetton. Stability analysis for the immersed fiber problem. SIAM Journal on Applied Mathematics, 55(6):1577-1591, 1995. 1, 2

[29] M. Sussman, P. Smereka, and Osher S. A level set approach for computing solutions to incompressible two-phase flow. Journal of Computational Physics, 114:146-159, 1994. 1

[30] Mark Sussman and Mitsuhiro Ohta. A stable and efficient method for treating surface tension in incompressible two-phase flow. SIAM Journal on Scientific Computing, 31(4):2447-2471, 2009. 1

[31] Cheng Tu and Charles S Peskin. Stability and instability in the computation of flows with moving immersed boundaries: a comparison of three methods. SIAM Journal on Scientific and Statistical Computing, 13(6):1361-1376, 1992. 1

[32] Jian-Jun Xu, Zhilin Li, John Lowengrub, and Hongkai Zhao. A level-set method for interfacial flows with surfactant. Journal of Computational Physics, 212(2):590-616, 2006. 1 